\title{
The ICARUS experiment
}

\author{
Christian Farnese ${ }^{1, a}$, On behalf of the ICARUS collaboration \\ ${ }^{1}$ Dep. of Physics and Astronomy G. Galilei, via Marzolo 8, 35131 Padova, Italy
}

\begin{abstract}
The 760 ton liquid argon ICARUS T600 detector performed a successful threeyear physics run at the underground LNGS laboratories, studying neutrino oscillations with the CNGS neutrino beam and searching for atmospheric neutrino interactions in cosmic rays. A sensitive search for LSND like anomalous $v_{e}$ appearance has been performed, contributing to constrain the allowed parameters to a narrow region around $\Delta m^{2} \sim e V^{2}$, where all the experimental results can be coherently accommodated at $90 \%$ C.L.. After a significant overhauling, the T600 detector will be exposed at Fermilab to the Booster Neutrino Beam acting as the far detector, in order to search for sterile neutrino within the SBN program. In the present contribution, the ICARUS LNGS achievements, the present status of the detector and the ongoing analyses also finalized to the next physics run at Fermilab will be addressed.
\end{abstract}

\section{The ICARUS T600 detector: a powerful detector for the neutrino physics}

The Liquid Argon Time Projection Chamber (LAr-TPC), an "effective electronic bubble-chamber" first proposed by C. Rubbia in 1977 [1], is a continuously sensitive and self triggering detector, that can provide a detailed 3D imaging and a precise calorimetric reconstruction of any ionizing event. The ICARUS T600 detector is the largest LAr-TPC detector ever built (476 t of LAr active mass) and it has been operated for 3 years in the Hall B of the Gran Sasso underground National Laboratory of Istituto Nazionale di Fisica Nucleare (INFN). The construction and successful operation of the T600 detector finalized many years of $R \& D$ studies made by the ICARUS Collaboration with the continuos support of the INFN: it represents the state of the art of this detection technique and the major milestone towards the realization of a multi-kiloton LAr-TPC detector.

The T600 consists of a large cryostat divided into two identical, adjacent modules. Each module houses two Time Projection Chambers made of three parallel wire planes, $3 \mathrm{~mm}$ apart, the first with horizontal wires and the other two at $\pm 60^{\circ}$ from the horizontal direction. By appropriate voltage biasing, the first two Induction planes faced to the drift region provide signals in non-destructive way and the charge is collected in the last Collection wire plane. The two TPCs in each module are separated by a common cathode and the maximal drift length (distance between the cathode and the wire planes), is $\sim 1.5 \mathrm{~m}$ corresponding to $\sim 1 \mathrm{~ms}$ drift time for the nominal drift field of $500 \mathrm{~V} / \mathrm{cm}$. Behind the wire planes, an array of PMTs (20 in West module and 54 in the East module) has been located and used for triggering purposes [2,3]. A more detailed description of the ICARUS T600 detector can be found in $[4,5]$.

a e-mail: christian.farnese@pd.infn.it 


\subsection{Liquid Argon purity achievements during the LNGS run}

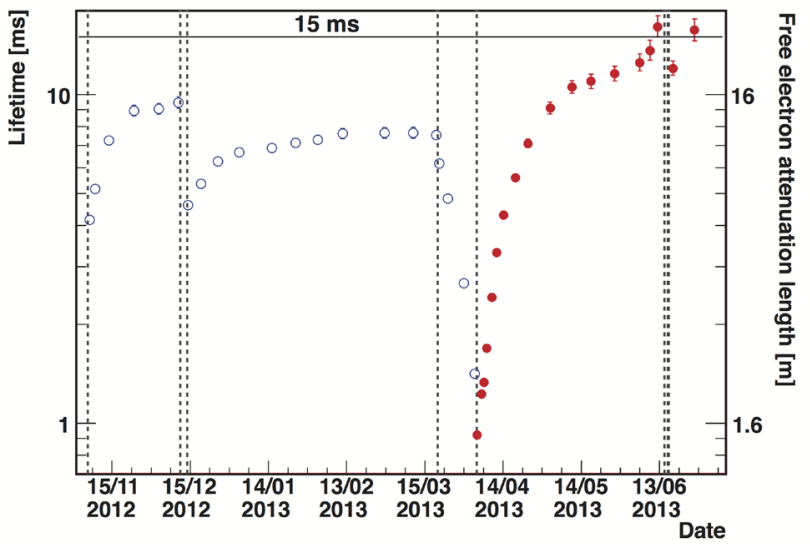

Figure 1. Electron life-time in the T600 East half-module, as a function of time [6]. The installation of a faster not immersed pump from April 2013 permitted to obtain a new minimum of impurity concentration, $<20 \mathrm{ppt} \mathrm{O}_{2}$ equivalent.

The main technological challenge of the development of the LAr-TPC is the capability to ensure long drift path for the ionization electrons. The presence of electron trapping polar impurities, most of all $\mathrm{O}_{2}$, in the Liquid Argon produces an exponential attenuation of the electron signal along the drift coordinate, that can result in a ionization signal indistinguishable from the wire noise. For this reason, the Argon must be filtered and recirculated forcing both the liquid and the gas phase through industrial chemical absorbers and molecular sieves. In addition the impurity level in the liquid must be continuously monitored measuring, on an event-by-event basis, the charge signal attenuation on the wires along cosmic-ray muon tracks crossing the LAr active volume. In this way it is possible to correct the attenuation effect and to obtain a better energy deposition measurement. The results on the LAr purity measurements during the Gran Sasso run show that an electron lifetime exceeding $7 \mathrm{~ms}$ ( $<50$ ppt $\mathrm{O}_{2}$ equivalent, $12 \%$ maximum charge attenuation at longest drift distance) has been obtained for the most part of the ICARUS data taking. Improvements in the recirculation system in the last months of the data taking allowed to reach an unprecendented $16 \mathrm{~ms}$ lifetime corresponding to $20 \mathrm{ppt} \mathrm{O}_{2}$ equivalent LAr contamination (Figure 1) [6], thus demonstrating the effectiveness of single-phase LAr-TPC technique and paving the way to the preparation of huge LAr-TPC detectors (up to $5 \mathrm{~m}$ drift).

\subsection{The T600 performance at LNGS}

The events collected during the Gran Sasso run demonstrate that the LAr-TPCs are very suitable detectors in particular for the study of rare events, such as neutrino oscillation physics and proton decay searches, because of the high spatial granularity (resolution of $\sim 1 \mathrm{~mm}^{3}$ on a overall active volume of $340 \mathrm{~m}^{3}$ for the ICARUS T600) and of the good homogeneous calorimetric response $\left(\sigma_{E} / E \approx 3 \% / \sqrt{E(G e V)}\right.$ for the e.m. showers and $\sigma_{E} / E \approx 30 \% / \sqrt{E(G e V)}$ for hadronic showers). 
In addition, the high density of sampling - corresponding to $\sim 2 \%$ of a radiation length - and the remarkable signal/noise ratio of $\sim 10 / 1$ on individual wires allow to measure in a very precise way the $\mathrm{dE} / \mathrm{dx}$ of each produced particle inside the detector providing the particle identification for stopping particles [7] and also a powerful electron $/ \gamma$ separation [8].

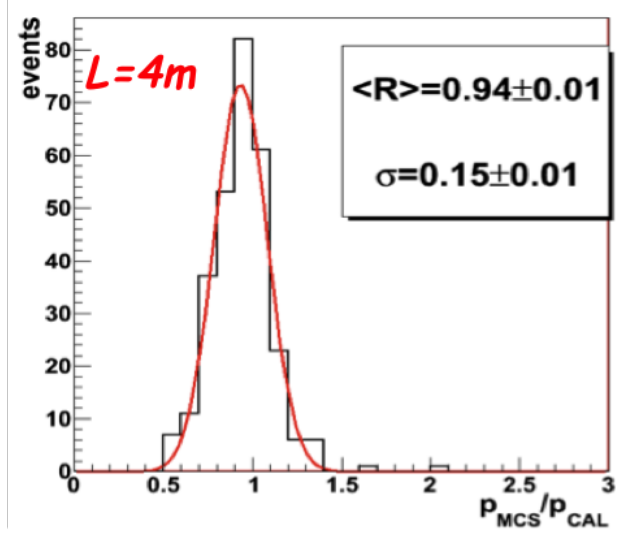

Figure 2. Distribution of the ratio between the stopping muon momentum obtained by MCS algorithm and the corresponding momentum evaluated by the calorimetric reconstruction.

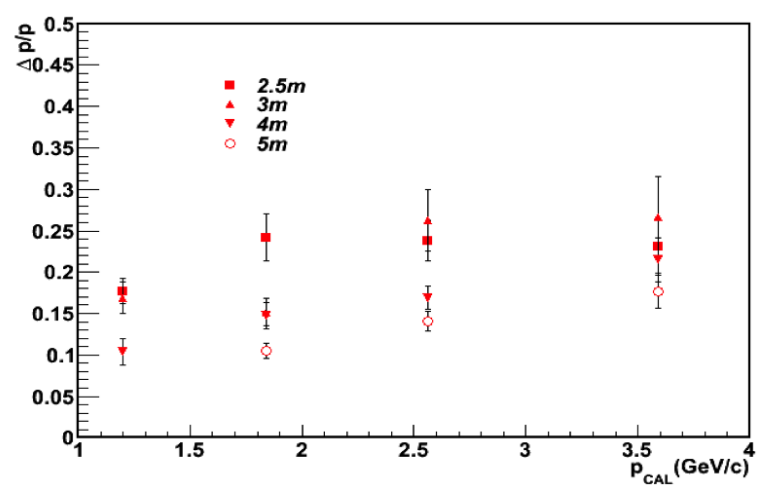

Figure 3. Dependency of momentum resolution on calorimetric muon momentum for different values of the used muon track length.

Momentum of escaping muons can be determined studying the multiple Coulomb scattering (MCS) along the collected track [9]. The measurement algorithm has been validated by using muons from CNGS $v_{\mu} C C$ interaction in the rock upstream of T600, stopping in the LAr volume; this represents an ideal validation sample, since the MCS momentum estimate $p_{M C S}$ can be compared directly with the calorimetric evaluation of the momentum $p_{C A L}$ (for this measurement the obtained resolution is $\Delta p / p \sim 1 \%)$. The comparison between the calorimetric momentum measurement and the result of 
the multiple scattering analysis $p_{M C S}$ is shown in figure 2. Some small underestimations have been observed for $\mathrm{p}>3.5 \mathrm{GeV} / \mathrm{c}$, due to the non-perfect planarity of the TPC cathode which introduces distorsions on the eletric drift field. After correcting for the $\sim \mathrm{cm}$ cathode distorsions, the MCS and calorimetric estimates of momentum agree on average within the errors, confirming the viability of the measurement. The momentum resolution obtained as a function of the different track length used for the analysis and of the initial muon momentum is also shown in figure 3: an average resolution $\Delta p / p \sim 15 \%$ is obtained in the $0.4-4 \mathrm{GeV} / \mathrm{c}$ energy range, which is relevant to the next generation neutrino experiments.

\subsection{LSND-like search by the ICARUS experiment at LNGS}

The possible existence of sterile neutrinos, particles that don't interact via any fundamental interactions of the Standard Model except gravity, has been firstly proposed by B. Pontecorvo [11]. Recent experiments have reported two distinct class of neutrino anomalies which, if confirmed, could hint at the presence of this additional neutrino flavour. First of all, an anomalous excess of $v_{e} / \bar{v}_{e}$ events has been observed in experiments using muon neutrino beams from particle accelerators (the socalled LSND and MiniBooNE anomalies $[12,13])$; at the same time additional $v_{e}$ or $\bar{v}_{e}$ disappearance anomalies have been reported in nearby nuclear reactors $[14,15]$ and Mega-Curie k-capture calibration sources in experiments dedicated to solar neutrinos $[16,17]$. All these anomalous $v_{e}$ and $\bar{v}_{e}$ evidences may imply the presence of an additional mass-squared difference somewhere within a wide interval $\Delta m_{\text {new }}^{2} \sim 0.01$ to $1.0 \mathrm{eV}^{2}$, largely in excess of the predictions of the Standard Model and three neutrino mixing. More recent experimental results still leave open questions: the IceCube detector keeps finding no evidence of muon neutrino disappearance in the $320 \mathrm{GeV}-20 \mathrm{TeV}$ energy range [18], while the results related to the electron antineutrinos from reactors provided by NEOS Collaboration can still be interpreted as evidence at $2.1 \sigma$ of short baseline oscillation [19]. On the other hand data from CMB experiments [20], large scale structures and Lyman- $\alpha$ forest observations bind total mass for 3 massless +1 massive sterile neutrinos to $\mathrm{m}<0.23 \mathrm{eV}$ at $95 \% \mathrm{CL}$, and should effectively exclude sterile neutrino as explanation of LSND anomaly.

The ICARUS Collaboration has performed a sensitive search for a possible $v_{e}$ excess in the CNGS $v_{\mu}$ beam related to the LSND hypothesis. From October 2010 to December 2012 ICARUS has collected neutrino interactions from the CNGS Cern to Gran Sasso neutrino beam corresponding to $8.6 * 10^{19}$ protons on target (POT) with a livetime exceeding $93 \%$. Neutrino events in the $10-30$ $\mathrm{GeV}$ energy range have been recorded with unprecedented details demonstrating the high-level technical performances and the physical potentialities of this novel detection technique. In particular the capabilities to reconstruct the neutrino interaction vertex, to identify and measure e.m. showers generated by primary electrons and to accurately measure the invariant mass of photon pairs allowed to provide an high efficiency in the identification of the electron-neutrino events and to reject to unprecedented level the NC background for the study of $v_{\mu} \rightarrow v_{e}$ transitions.

In total $2650 \mathrm{CNGS}$ neutrino interactions have been identified in $7.910^{19}$ pot statistics, $\sim 3.4$ neutrino interactions and $\sim 12$ beam related muons per $10^{17}$ p.o.t., consistent within $6 \%$ with $\mathrm{MC}$ predictions. These neutrino events have been studied in details and globally 7 electron-like events have been observed to be compared with the $8.5 \pm 1.1$ expected from the $\sim 1 \%$ intrinsic beam component and standard 3-flavor oscillations, providing the limit on $v_{\mu} \rightarrow v_{e}$ oscillations $P<3.8510^{-3}$ at $90 \% \mathrm{CL}$ and $P<7.6010^{-3}$ at $99 \%$ CL (Fig. 4) $[8,10]$. 


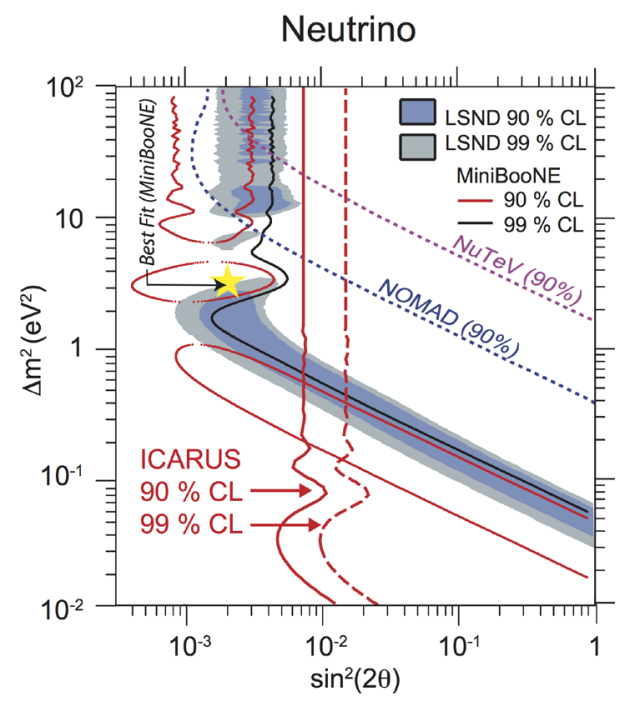

Figure 4. ICARUS-T600 exclusion plot at 90 and $99 \% \mathrm{CL}$ on the $v_{\mu} \rightarrow v_{e}$ LSND-like oscillation search at CNGS beam compared to LSND allowed region. Other experimental limits are also shown.

\subsection{Atmospheric neutrino search in the T600 detector}

The T600 detector collected also events related to cosmic rays in order to study the detector capability for the atmospheric neutrinos and proton decay search. In particular cosmic ray events recorded in $\sim 0.48$ kton y exposure (2012-2013 run) are being analysed to identify and study atmospheric neutrino events, since they cover the energy range expected for the future SBN experiment at FNAL (see Sect. 2). A fully automatic algorithm has been set up in order to reject the incoming cosmic ray tracks and to select neutrino candidates, providing a $\sim 70 \%$ efficiency for electron neutrino events and a factor $\sim 100$ rejection for the through-going cosmic muons. The neutrino candidates are then validated by visual scanning, to select the genuine neutrino interactions.

About $\sim 50 \%$ of the selected exposure has been analyzed so far and $7 v_{\mu} \mathrm{CC}$ and $8 v_{e} \mathrm{CC}$ atmospheric neutrino events have been identified and reconstructed, demonstrating as the automatic search for the $v_{e} \mathrm{CC}$ in the sub-GeV range, required for the study of the BNB neutrinos at FNAL, is feasible. The results of the reconstruction of 3 identified neutrino events are shown in figures 5, 6 and 7.

\section{Moving forward: the new Short Baseline Neutrino program at FNAL}

A conclusive experiment, capable of clarifying at $5 \sigma$ level the nature of the LSND anomaly and the possible existence of sterile neutrinos (see Sect. 1.3), is still needed. This new project should be based on two innovative concepts: the use of LAr-TPC technology, which provides excellent tracking and reconstruction capabilities, and the use of multiple stations measuring the neutrino spectrum at different distances from the target: oscillation signals would be identified as differences in the measured spectra while identical spectra would imply no oscillatory behaviour, helping in addition in the elimination of the systematics effects. Such a program is now under preparation at the Fermi National Accelerator Laboratory: the SBN short-baseline experimental configuration will involve 

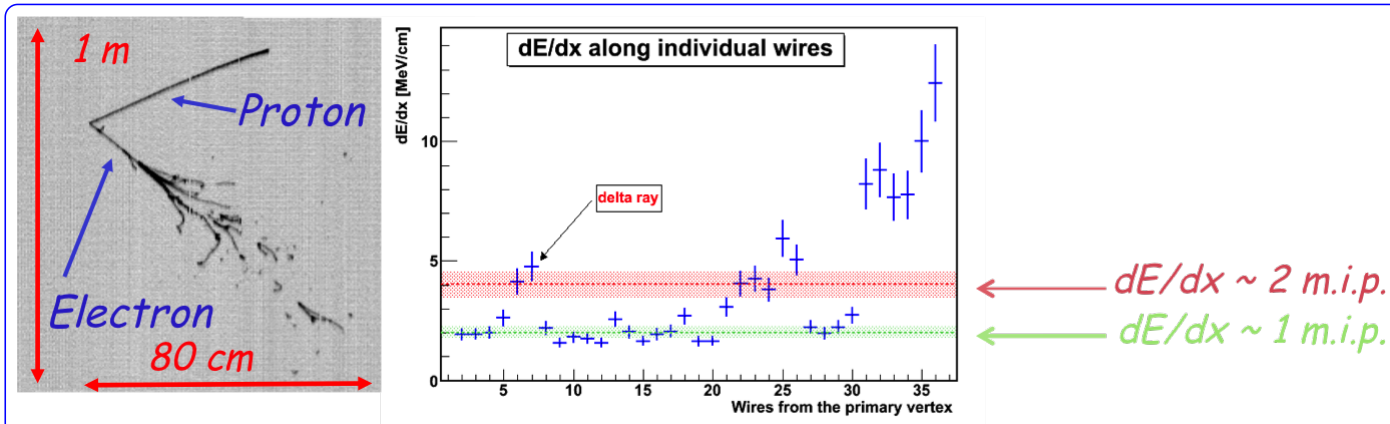

Figure 5. Atmospheric electron neutrino event with a deposited energy of $0.9 \mathrm{GeV}$ is shown in the 2D Collection view of ICARUS-T600 (left). The evolution of the ionization density $\mathrm{dE} / \mathrm{dx}$ in the first wires of the shower is also shown (right), providing a clear signature for the electron identification. A proton track has been also identified studying the $\mathrm{dE} / \mathrm{dx}$ versus range relation.
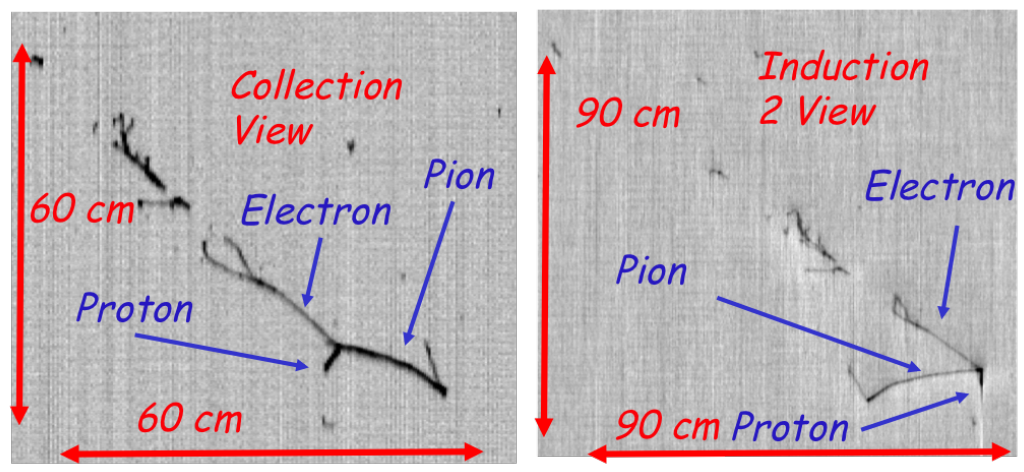

Figure 6. Downward- going atmospheric electron neutrino event with a deposited energy of $0.42 \mathrm{GeV}$ is shown in the 2D Collection (left) and Induction 2 (right) views of ICARUS-T600. An electron with a deposited energy $E_{\text {dep }} \sim 240 \mathrm{MeV}$, a proton $\left(E_{\text {dep }} \sim 60 \mathrm{MeV}\right)$ and a pion $\left(E_{\text {dep }} \sim 120 \mathrm{MeV}\right)$ have been recognized. A preliminary reconstruction of the neutrino direction provides a zenith angle $\sim 108^{\circ}$.

three LAr-TPC detectors (SBND, MicroBooNE and ICARUS-T600) installed on axis in the BNB neutrino beamline at $110 \mathrm{~m}, 470 \mathrm{~m}$ and $600 \mathrm{~m}$ distance from target respectively.

This program, fully described in [21], will allow a very sensitive search for high- $\Delta m^{2}$ neutrino oscillations in multiple channels, accumulating $6.6 \cdot 10^{20}$ protons on target in 3 years. The expected $v_{e} \mathrm{CC}$ event distributions expected in the Near and Far detectors with an oscillation signal corresponding to the best-fit parameters from the analysis of $\Delta m^{2}=0.43 \mathrm{eV}^{2}$ and $\sin ^{2}(2 \theta)=0.013$ are shown in figure 8 , including also all the expected backgrounds.

The resulting experimental sensitivity of the SBN program to $v_{\mu} \rightarrow v_{e}$ appearance signals in the $\left(\Delta m^{2}, \sin ^{2}(2 \theta)\right)$ plane is shown in figure 9: the LSND 99\% C.L. allowed region is covered at the $\sim 5 \sigma$ level everywhere; the region below $\Delta m^{2}=0.1 \mathrm{eV}^{2}$ has been already ruled out at more than $5 \sigma$ by the previous results of ICARUS at Gran Sasso. Since a genuine $v_{\mu} \rightarrow v_{e}$ appearance can also be accompanied by a disappearance of the intrinsic $v_{e}$ beam component, the disappearance measurement 


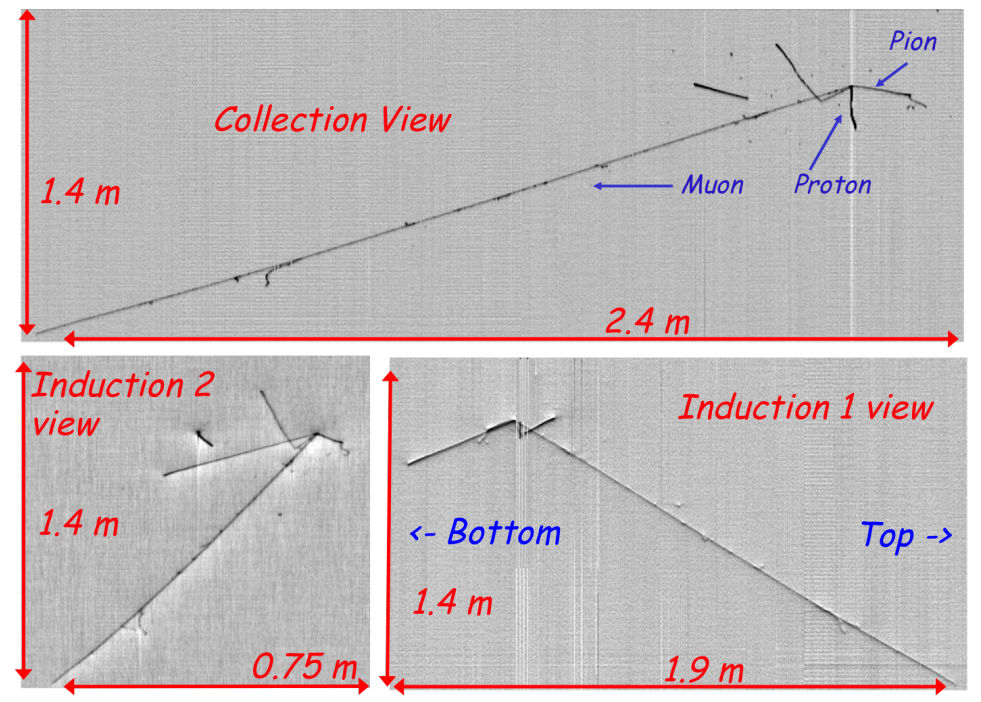

Figure 7. A clear upward-going atmospheric muon neutrino event with a deposited energy of $1.7 \mathrm{GeV}$ is shown in the 3 different views of ICARUS-T600. The momentum of the $4 \mathrm{~m}$ escaping muon has been evaluated using the MCS algorithm, providing $\mathrm{p}=1.8 \pm 0.3 \mathrm{GeV} / \mathrm{c}$. Three hadrons are produced in the neutrino interaction vertex, two of which has been identified as a proton with a deposited energy $E_{d e p} \sim 250 \mathrm{MeV}$ and a pion decaying in a muon followed by an electron $\left(E_{\text {dep }} \sim 80 \mathrm{MeV}\right)$. A preliminary global reconstruction of the neutrino interaction provides $E_{v} \sim 2 \mathrm{GeV}$, with a zenith angle $\sim 78^{\circ}$.

is needed in the SBN program to confirm a signal, if seen in $v_{e}$ appearance, as oscillations. The ability to perform searches for oscillation signals in multiple channels is a major advantage for the FNAL SBN oscillation physics program. The simultaneous analysis of $v_{e} \mathrm{CC}$ and $v_{\mu} \mathrm{CC}$ events in the same experiment will be a very powerful way to explore oscillations and disentangle the effects of $v_{\mu} \rightarrow v_{e}$, $v_{\mu}$ disappearance, and $v_{e}$ disappearance, if they exist, in this mass-splitting range. Even if the absolute neutrino flux and cross section uncertainties in any detector are expected to be larger than $10 \%$, the high correlations between the near detector and the MicroBooNE/ICARUS event samples combined with the excellent statistics at the near site will make the SBN program the most sensitive $v_{\mu}$ disappearance experiment at $\Delta m^{2} \sim 1 \mathrm{eV}^{2}$ (figure 9 right).

During the data taking at Fermilab, the T600 detector will also exploit, in parallel to the Booster neutrino events, the off-axis neutrinos from the NuMI beam, collecting a large statitistics comparable to the one from the Booster beam, in the $0 \div 3 \mathrm{GeV}$ energy range with an enriched component of electron neutrinos (several \%) from the dominant three body decay of secondary $K$. A careful and detailed analysis of these events will allow to study very precisely detection efficiencies, neutrino cross-sections and interaction topologies at energies relevant to the future Long Baseline Neutrino Facility (LBNF) program with the multi-kt DUNE LAr-TPC detector [22].

\section{Overhauling the T600 detector at CERN}

In order to prepare the detector for this new experimental phase, the T600 detector has been transported to CERN and, starting from December 2014, the T600 underwent a significant overhauling 

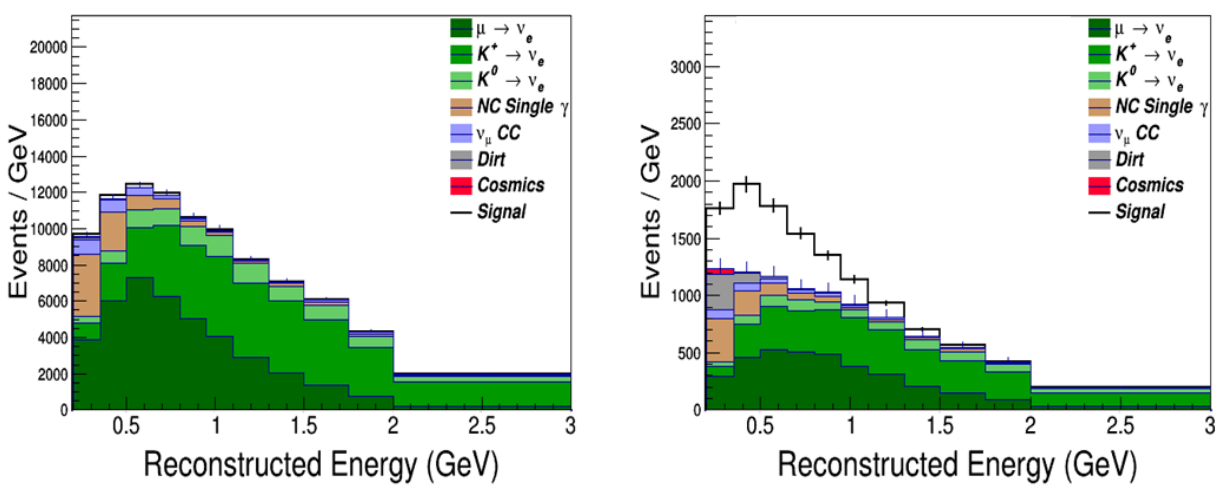

Figure 8. The expected $v_{e} \mathrm{CC}$ event distribution at near SBND (left) and far ICARUS-T600 (right) detectors for a $6.6 \cdot 10^{20}$ pot exposure at BNB beam including also $v_{e}$ beam events, misidentified neutral-current and $v_{\mu}$ $\mathrm{CC}$ events, out-of-detector beam related backgrounds, and cosmogenic photon induced electromagnetic shower backgrounds. The $v_{\mu} \rightarrow v_{e}$ signal refers to $\Delta m^{2}=0.43 \mathrm{eV}^{2}$ and $\sin ^{2} 2 \theta_{\mu e}=0.013$.
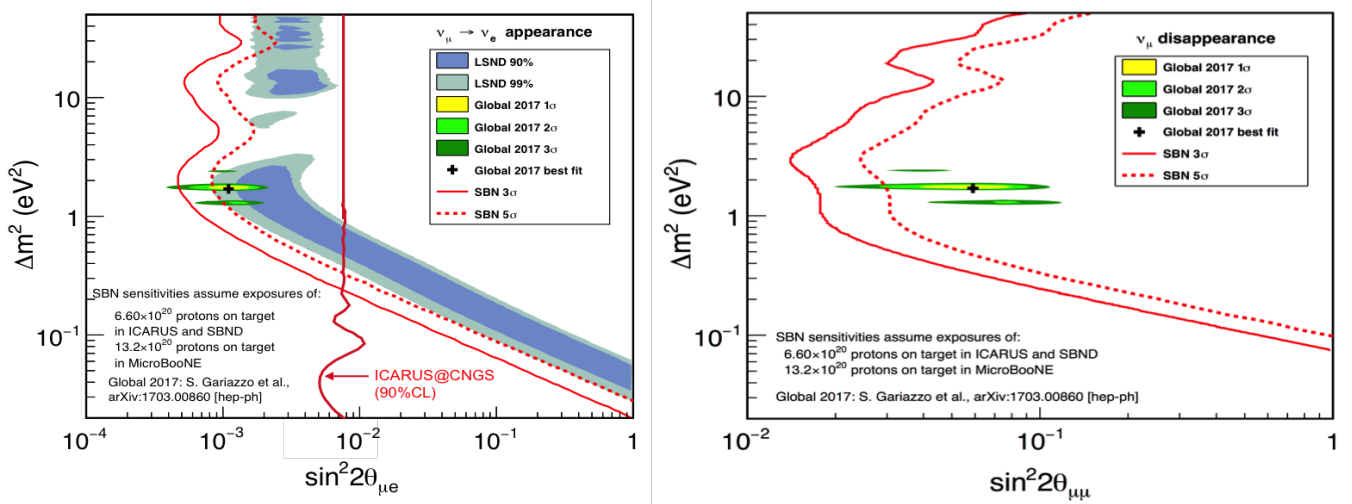

Figure 9. The expected SBN sensitivity to $v_{\mu} \rightarrow v_{e}$ (left) and the corresponding expected sensitivity to $v_{\mu}$ disappearance (right) for $6.6 \cdot 10^{20}$ pot exposure at BNB beam (twice for MicroBooNE), compared to LSND positive result and to current global best fit expectation of all present neutrino anomalies [23].

phase in order to introduce some technology developments while maintaining the already achieved performances. The most important activities in the framework of CERN Neutrino Platform (WA104 project) concern:

- a new, higher-performance read-out electronics: the adoption of serial 2-bit ADCs, one per channel, in place of the multiplexed ones used in T600 at LNGS provides a faster read-out system, a synchronous sampling (400 ns) of all channels of the whole detector and a very compact set-up, 
allowing for hosting both analog and digital electronics directly on the feed-through flanges; a more detailed description of this new system and of the tests done to validate it can be found in a poster contribution at this conference [24];

- the realisation and installation of a new scintillation light detection system: 360 Hamamatsu R5912MOD PMTs have been first of all tested at room temperature, made sensitive to ultraviolet photons by coating their windows with a proper fluorescent wavelength shifter and finally installed behind the 4 wire chambers (90 units each); 60 units were also characterized at cryogenic temperature, in a liquid argon bath, to verify any feature variation, such as the PMT gain, dark counts and saturation. This improved system will provide an high detection coverage and granularity, to be able to localise also low energy deposition in the TPC $(\sim 100 \mathrm{MeV})$, and it will also be characterised by a high time resolution $(\sim 1 \mathrm{~ns})$ to exploit the bunched structure of the Booster Neutrino beam and to be sensitive to the evolution in time of each event collected in the LAr active volume.

- the construction of new cold vessels, made of extruded aluminum profiles welded together, and of the new passive insulation for the detector;

- the refurbishment of the cryogenics and LAr purification system;

- the flattening of the TPC cathode panels: the presence of intrinsic deformations during the Gran Sasso run (25 mm maximal deviation from flatness) produced electric field distortions large enough to hamper precision physics measurements, like the muon momentum evaluation based on MCS analysis; for this reason, the cathode panels underwent thermal treatment that provide a reduction of the deformations by a factor 10 ;

The T600 detector at Fermilab should also face more challenging experimental conditions with respect to the deep underground conditions of the LNGS laboratory: in the previous run a single prompt trigger has always ensured the unique timing connection of the main image of the event which determine the track position along the drift coordinate. At Fermilab the T600 will take data at shallow depth, protected only by a $3 \mathrm{~m}$ concrete overburden: even if this shielding results to be enough to suppress the cosmic rays hadronic component and to reduce cosmic muons by $\sim 30 \%, \sim 11$ additional cosmic muons are expected to cross the detector randomly in the $1 \mathrm{~ms}$ drift time corresponding to the triggered event, introducing some difficulties for the event track reconstruction. In addition the photons produced by the crossing muons represent a serious source of background for the electron neutrino search since the electrons produced by the Compton scattering and by the pair production processes can mimic a genuine $v_{e} \mathrm{CC}$ interaction. In order to mitigate this important source of background, a $4 \pi$, segmented cosmic muon tagging system (CTS) composed by plastic scintillation slabs read out by silicon PMTs surrounding the T600 (area $~ 1200 \mathrm{~m}^{2}$, coverage $\sim 98 \%$ ) will be installed. The information provided by this system, combined with the signals from the internal light detection system, will allow to unambiguously identify the cosmic ray tracks entering the detector and will help to select the genuine neutrino interactions.

The T600 overhauling process has been concluded in June 2017 with the installation of the two T600 internal detectors, completely refurbished, in the new aluminum vessels. The two ICARUS module has been then transported to FNAL and safely arrived at the far site of the SBN experiment on July 26, 2017. The installation and commissioning will follow, to start as soon as possible the data taking on the Booster Neutrino Beam, to definitively clarify the "sterile neutrino puzzle".

\section{References}

[1] C. Rubbia, CERN-EP/77-08 (1977). 
[2] A. Ankowski et al. (ICARUS Coll.), Nucl. Instr. and Meth. A556 149 (2005).

[3] M. Antonello et al. (ICARUS coll.), JINST 9 P08003 (2014)

[4] S. Amerio et al. (ICARUS Coll.), Nucl. Instr. and Meth. A 527, 329 (2004).

[5] C. Rubbia et al.(ICARUS Coll.), JINST 6 P07011 (2011)

[6] M. Antonello et al.(ICARUS Coll.), JINST 9 P12006 (2014)

[7] M. Antonello et al. (ICARUS Coll.), Adv. High Energy Phys., 260820 (2013).

[8] M. Antonello et al. (ICARUS Coll.), Eur. Phys. J. C, 732345 (2013).

[9] M. Antonello et al.(ICARUS Coll.), JINST 12 P04010 (2017)

[10] M. Antonello et al., Eur. Phys. J. C 732599 (2013).

[11] B. Pontecorvo, Zh. Eksp. Teor. Fiz. 53, 1717 (1967) [Sov. Phys. JETP 26, 984 (1968)].

[12] A. Aguilar et al. (LSND Coll.), Phys. Rev. D 64, 112007 (2001).

[13] A. A. Aguilar-Arevalo et al. (MiniBooNE Coll.), Phys. Rev. Lett. 110, 161801 (2013) and references therein.

[14] G. Mention et al., Phys. Rev. D 83 (2011) 073006 and references therein.

[15] C. Zhang, X Quian and P. Vogel, Phys. Rev. D 87 (2013) 073018.

[16] J. N. Abdurashitov et al (SAGE Coll.), Phys. Rev. C 80, 015807 (2009).

[17] F. Kaether, W. Hampel, G. Heusser, J. Kiko, and T. Kirsten, Phys. Lett. B 47, 685 (2010) and references therein.

[18] Aartsen M.G. et al. (IceCube Collab.), Phys. Rev. Lett. 117, 071801 (2016)

[19] Ko Y.J. et al. (NEOS Collab.), Phys. Rev. Lett. 118, 121802 (2017)

[20] Planck Collaboration, Astron.Astrophys, 594, A13 (2016)

[21] R. Acciarri et al., Preprint arXiv:1503.01520 (2015).

[22] R. Acciarri et al., Preprint arXiv:1601.05471 (2016).

[23] S. Gariazzo et al., JHEP 06135 (2017)

[24] C. Farnese, The new front end and DAQ of the ICARUS detector, these proceedings (2017) 